Communications in Physics, Vol. 29, No. 1 (2019), pp. 87-96

DOI:10.15625/0868-3166/29/1/13556

\title{
LEPTON FLAVOR VIOLATION IN ECONOMICAL 3-3-1 MODEL WITH NEUTRINO SINGLETS
}

\author{
LE THO HUE ${ }^{1}$ DINH PHAN KHOI ${ }^{2}$, HOANG HANH PHUONG $^{3}$ AND HA THANH HUNG ${ }^{3, \dagger}$ \\ ${ }^{1}$ Institute of Physics, Vietnam Academy of Science and Technology, \\ 10 Dao Tan, Ba Dinh, Hanoi, Vietnam \\ ${ }^{2}$ Department of Physics, Vinh University, 182 Le Duan, Vinh City, Nghe An, Vietnam \\ ${ }^{3}$ Department of Physics, Hanoi Pedagogical University 2, Phuc Yen, Vinh Phuc, Vietnam \\ ${ }^{\dagger}$ E-mail: hathanhhung@hpu2.edu.vn
}

Received 15 January 2019

Accepted for publication 06 March 2019

Published 15 March 2019

\begin{abstract}
Lepton flavor violating (LFV) decays of charged leptons $\mu \rightarrow$ e $\gamma$ and SM-like Higgs boson $h \rightarrow \mu \tau$ are discussed in the framework of the economical 3-3-1 model adding three additional singlets neutrinos $\left(E 331 X_{R}\right)$. We will show that the appearance of new neutrinos and charged Higgs bosons in this model can result in interesting regions of the parameter space, which satisfy the recent experimental bound of $\mathrm{Br}(\mu \rightarrow e \gamma)$ as well as give large $\operatorname{Br}(h \rightarrow \mu \tau) \geq \mathscr{O}\left(10^{-7}\right)$. In these regions, the charged Higgs boson mass lies within the range of $2-3 \mathrm{TeV}$. Our results show that the LFV decays are important channels to distinguish the E331X $X_{R}$ and the original economical (E331) models, which predict suppressed LFV decay rates.
\end{abstract}

Keywords: electroweak interactions, models beyond the Standard Model, non-standard-model neutrinos, right-handed neutrinos.

Classification numbers: 12.15.-y, 12.60.-i,14.60.St. 


\section{INTRODUCTION}

The economical 331 models $[1,2]$ are the simpler versions of the model with right-handed neutrino (331RN) [3], where only two Higgs triplets are introduced to generate masses of particles. These E331 models predict new neutrinos with very light masses, result in suppressed LFV processes like $\mu \rightarrow e \gamma$ and $h \rightarrow \mu \tau$, which are now being searched experimentally as signal of new physics beyond the Standard Model (SM) [4,5]. Recently, new versions of the 331RN, where new singlet neutrinos are included, predicted heavy neutrinos through the inverse seesaw (ISS) mechanism [6,7]. These models also predict that the $\operatorname{Br}(\mu \rightarrow e \gamma)$ can reach the recent experimental bound, and the LFV decay of the SM-like Higgs boson $\operatorname{Br}(h \rightarrow \mu \tau)$ may be large [7]. In this work, we will investigate the two mentioned LFV decays in the E331 $X_{R}$. Because of the simpler Higgs sectors than the previous 3-3-1 versions with ISS neutrinos, the LFV signal predicted by the E331 $X_{R}$ can not be estimated based on the previous investigations. Therefore a detailed discussion in this work is very important and useful.

Our paper is arranged as follows. In Sec. II, we review ingredients of the E331 model relevant with the LFV processes. After that, we focus on important content of the E331 $X_{R}$, including ISS mechanism for generating neutrino masses, LFV couplings, and branching ratio of the two decays $\mu \rightarrow e \gamma$ and $h \rightarrow \mu \tau$. In Sec. III, numerical illustrations are presented. Section IV is our inclusion, where main results are given.

\section{THE ECONOMICAL 3-3-1 MODEL WITH THREE NEUTRINO SINGLETS}

\section{II.1. The original E331 model}

The economical 3-3-1 model (E331) was constructed based on the gauge group $S U(3)_{C} \times$ $S U(3)_{L} \times U(1)_{X}$ with two scalar triplets [1,2]. We review here important

$$
\rho=\left(\begin{array}{c}
\rho_{1}^{+} \\
\rho_{2}^{0} \\
\rho_{3}^{-}
\end{array}\right) \sim\left(3, \frac{2}{3}\right) ; \quad \phi=\left(\begin{array}{c}
\phi_{1}^{0} \\
\phi_{2}^{-} \\
\phi_{3}^{0}
\end{array}\right) \sim\left(3, \frac{-1}{3}\right)
$$

with vacuum expectation values (VEVs) are $\left\langle\phi_{1}^{0}\right\rangle=u / \sqrt{2},\left\langle\phi_{3}^{0}\right\rangle=w / \sqrt{2}$, and $\left\langle\rho_{2}^{0}\right\rangle=v / \sqrt{2}=174$ $\mathrm{GeV}$. It is enough to consider here $u=0$, where the neutrinos masses will arise from the ISS mechanism.

Apart from photon, the E331 contains eight massive gauge bosons after spontaneous breaking, namely four neutral bosons $\left(X^{0}, X^{0 *}\right),\left(Z, Z^{\prime}\right)$ and four singly charged ones $\left(W^{ \pm}, V^{ \pm}\right)$. The masses used in this work are $m_{W}=\frac{g v}{2} ; m_{X}=\frac{g w}{2} ; m_{V}=\frac{g \sqrt{v^{2}+w^{2}}}{2}$. The $S U(3)_{L}$ scale predicts new heavy particles, leading to the condition $w \gg v$.

The Higgs potential with only two scalar triplets is

$$
\mathscr{V}_{\mathrm{H}}=\mu_{1}^{2} \phi^{\dagger} \phi+\mu_{2}^{2} \rho^{\dagger} \rho+\lambda_{1}\left(\phi^{\dagger} \phi\right)^{2}+\lambda_{2}\left(\rho^{\dagger} \rho\right)^{2}+\lambda_{3}\left(\phi^{\dagger} \phi\right)\left(\rho^{\dagger} \rho\right)+\lambda_{4}\left(\rho^{\dagger} \phi\right)\left(\phi^{\dagger} \rho\right) .
$$

The E331 has a simple physical Higgs spectrum: two charged Higgs boson $H_{2}^{ \pm}$, two CP-even Higgs $(h, H)$ and one CP-odd boson $A$. Their masses and mixing are given in detailed in Ref [2], where the Higgs boson $h$ is identified with that found by LHC. It is named the Standard Model-like (SM-like) Higgs boson because it has the similar property of the neutral Higgs boson predicted by 
the SM. The masses of Higgs bosons relevant with this work are:

$$
m_{h}^{2}=v^{2}\left[\left(2 \lambda_{2}-\frac{\lambda_{3}}{2 \lambda_{1}}\right)+O\left(\frac{v^{2}}{w^{2}}\right)\right], \quad m_{H_{2}^{ \pm}}^{2}=\frac{\lambda_{4}}{g}\left(m_{W}^{2}+m_{X}^{2}\right),
$$

In the E331 model, neutral leptons are all included in triplets, $\psi_{a L}=\left(v_{a}, e_{a}, N_{a}\right)_{L}^{T}, a=1,2,3$. The SM-like neutrino is $v_{a}$, and the new ones predicted by the E331 are $N_{a L}$. The lepton masses in the E331 model come from the Yukawa part

$$
\mathscr{L}_{l}^{\mathrm{Y}}=-h_{a b}^{e} \overline{\psi_{a L}} \rho e_{b R}+h_{a b}^{v} \epsilon^{i j k} \overline{\left(\psi_{a L}\right)_{i}}\left(\psi_{b L}\right)_{j}^{c} \rho_{k}^{*}+\text { h.c. }
$$

It was shown that the neutrinos in this model are all very light. Hence they result in very suppressed signals of LFV processes, which can not be detected by future experiments. In the next section, we will discuss the model containing heavy neutrinos.

In this work, we just focus on the decays relating with SM-like Higgs bosons and leptons, which are irrelevant with strong interactions like the quarks. The quark sector of the E331 model was discussed in detail previously [2], hence we will not repeat again. As we will see, the E331 $X_{R}$ model is different from the E331 model by the lepton sector. Therefore, in the following it will be constructed thoroughly for determining the LFV couplings.

\section{II.2. The $\mathbf{E 3 3 1}_{R}$ model}

In this work, the $\mathrm{E} 331 X_{R}$ is constructed by adding three new singlets of right-handed neutrinos $X_{a R} \sim(1,1,0), \mathrm{a}=1,2,3$. The new Lagrangian part different from the E331 model is

$$
-\mathscr{L}_{X_{R}}=Y_{a b} \overline{\psi_{a L}} \phi X_{b R}+\frac{1}{2}\left(\mu_{X}\right)_{a b} \overline{\left(X_{a R}\right)^{c}} X_{b R}+\text { h.c.. }
$$

Lagrangian (5) plays very important role to create masses and mixing of neutrinos following the ISS mechanism at tree level.

It can be seen that the $\mathrm{E} 331 X_{R}$ has total nine neutrinos including three SM-like light $v_{a}$, three exotic neutrinos at the bottom of lepton triplets $N_{a}$ and three right-handed neutrinos $X_{a R}$.

To determine neutrino masses and mixing, we will use the basis $v_{L}^{\prime}=\left(v_{L}, N_{L},\left(X_{R}\right)^{c}\right)^{T}$ and $\left(v_{L}^{\prime}\right)^{c}=\left(\left(v_{L}\right)^{c},\left(N_{L}\right)^{c}, X_{R}\right)^{T}$ with $v_{R}=\left(\left(v_{1 L}\right)^{c},\left(v_{2 L}\right)^{c},\left(v_{3 L}\right)^{c}\right)^{T}, N_{R}=\left(\left(N_{1 L}\right)^{c},\left(N_{2 L}\right)^{c},\left(N_{3 L}\right)^{c}\right)^{T}$, and $X_{L}=\left(\left(X_{1 R}\right)^{c},\left(X_{2 R}\right)^{c},\left(X_{3 R}\right)^{c}\right)^{T}$. Combining two Yukawa parts (4) and (5), the neutrino mass term is given as

$$
-\mathscr{L}_{\text {mass }}^{v}=\frac{1}{2} \overline{v_{L}^{\prime}} M^{v}\left(v_{L}^{\prime}\right)^{c}+\text { h.c. },
$$

where $M^{v}$ is the $9 \times 9$ matrix that is written in the block form as follows

$$
M^{v}=\left(\begin{array}{ccc}
0 & m_{D} & 0 \\
m_{D}^{T} & 0 & M_{R} \\
0 & M_{R}^{T} & \mu_{X}
\end{array}\right) .
$$

In the expression (7), $M_{R}$ is a $3 \times 3$ matrix having $\left(M_{R}\right)_{a b} \equiv Y_{a b} \frac{w}{\sqrt{2}}$ with $a, b=1,2,3, \mu_{X}$ is also a $3 \times 3$ matrix was derived in (5), $m_{D}$ is the Dirac mass matrix of neutrinos, which can be calculated in the same way shown in Ref. [7],

$$
m_{D}=z\left(\begin{array}{ccc}
0 & x_{12} & x_{13} \\
-x_{12} & 0 & 1 \\
-x_{13} & -1 & 0
\end{array}\right)
$$


Here, $z, x_{12}, x_{13}$ are independent parameters. The mass matrix (7) has the form which creates neutrino mass from the ISS mechanism.

The mixing matrix of neutrino $U^{v}$ satisfies the following condition

$$
U^{v T} M^{v} U^{v}=\hat{M}^{v}=\operatorname{diag}\left(\hat{m}_{v}, \hat{M}_{N}\right),
$$

where $\hat{m}_{v}=\operatorname{diag}\left(m_{n_{1}}, m_{n_{2}}, m_{n_{3}}\right)$ and $\hat{M}_{N}=\operatorname{diag}\left(m_{n_{4}}, m_{n_{5}}, \ldots, m_{n_{9}}\right)$, corresponding to the masses of active neutrinos and six extra neutrinos. The ISS mechanism guarantees that $m_{n_{a}}$ are light and can be fitted with the current neutrino data. On the other hand, six exotic neutrino masses have order of $\left|M_{R}\right| \sim w$, hence they are very heavy.

\section{II.3. Relevant LFV couplings and decays}

The LFV coupling relating with gauge bosons can be traced from previous work,

$$
\begin{aligned}
\mathscr{L}^{\ell \ell V} & =\overline{\psi_{a L}} \gamma^{\mu} D_{\mu} \psi_{a L} \\
& =\frac{g}{\sqrt{2}}\left[U_{a i}^{\nu *} \overline{e_{a}} \gamma^{\mu} P_{L} n_{i} W_{\mu}^{-}+U_{a i}^{v} \overline{n_{i}} \gamma^{\mu} P_{L} e_{a} W_{\mu}^{+} U_{(a+3) i}^{\nu^{*}} \overline{e_{a}} \gamma^{\mu} P_{L} n_{i} Y_{\mu}^{-}+U_{(a+3) i}^{v} \overline{n_{i}} \gamma^{\mu} P_{L} e_{a} Y_{\mu}^{+}\right]
\end{aligned}
$$

The LFV couplings relating with Higgs bosons are changed because of the new Yukawa couplings (5). The Yukawa part (4) gives

$$
\begin{aligned}
& -h_{a b}^{e} \overline{\psi_{a L}} \rho e_{b R}+h_{a b}^{v} \epsilon^{i j k} \overline{\left(\psi_{a L}\right)_{i}}\left(\psi_{b L}\right)_{j}^{c} \rho_{k}^{*}+\text { h.c. } \\
& \supset-\frac{g m_{a}}{\sqrt{2} m_{W}}\left[\frac{c \xi}{\sqrt{2}} h \overline{e_{a}} e_{a}+c_{\alpha}\left(U_{(a+3) i}^{v} \overline{n_{i}} P_{R} e_{a} H_{2}^{+}+U_{(a+3) i}^{v *} \overline{e_{a}} P_{L} n_{i} H_{2}^{-}\right)\right] \\
& -\frac{g c_{\xi}}{2 m_{W}} h\left[\sum_{c=1}^{3} U_{c i}^{v} U_{c j}^{v *} \overline{n_{i}}\left(m_{n_{i}} P_{L}+m_{n_{j}} P_{R}\right) n_{j}\right]-\frac{g c_{\alpha}}{\sqrt{2} m_{W}}\left[U_{a j}^{v *} m_{n_{j}}\left(U_{j(b+3)}^{v \dagger} U_{b i}^{v}\right) H_{2}^{-} \overline{e_{a}} P_{R} n_{i}+\text { h.c. }\right],
\end{aligned}
$$

where two mixing parameters $\alpha$ and $\xi$ are defined as

$$
t_{\alpha}=\frac{m_{W}}{m_{X}}=\frac{v}{w} ; \quad t_{2 \xi}=\frac{\lambda_{3} m_{X} m_{W}}{\lambda_{1} m_{X}^{2}-\lambda_{2} m_{W}^{2}} .
$$

In (5), the couplings from first term,

$$
\begin{array}{rr}
Y_{a b} \overline{\psi_{a L}} \phi X_{b R}+\text { h.c. } \\
\frac{g}{2 m_{W}}\left(M_{R}\right)_{a b}\left[h s_{\xi} t_{\alpha} U_{(a+3) i}^{v} U_{(b+6) j}^{v} \overline{n_{i}} P_{R} n_{j}-\left(\sqrt{2} c_{\alpha} t_{\alpha}^{2} U_{(b+6) i}^{v} \overline{e_{a}} P_{R} n_{i} H_{2}^{-}+\text {h.c. }\right)\right] .
\end{array}
$$

For convenience, we define

$$
\begin{array}{rr}
\lambda_{i j}^{0} & =\sum_{c=1}^{3}\left(U_{c i}^{v} U_{c j}^{\nu *} m_{n_{i}}+U_{c i}^{\nu *} U_{c j}^{v} m_{n_{j}}\right)-\sum_{c, d=1}^{3} t_{\alpha} t_{\xi}\left(M_{R}^{*}\right)_{c d}\left[U_{(c+3) i}^{\nu *} U_{(d+6) j}^{\nu *}+U_{(c+3) j}^{\nu *} U_{(d+6) i}^{\nu *}\right], \\
\lambda_{a i}^{R} & = \\
m_{a} U_{(a+3) i}^{v}, & \lambda_{a i}^{L}=\sum_{c=1}^{3}\left(m_{D}^{*}\right)_{a c} U_{c i}^{\nu *}+t_{\alpha}^{2}\left(M_{R}^{*}\right)_{a c} U_{(c+6) i}^{\nu *} .
\end{array}
$$

Based on the above calculation, all LFV couplings are listed in Table 1.

The partial decay width of the SM-like Higgs boson decay $h \rightarrow e_{a} e_{b}$ is $[8,9]$

$$
\Gamma\left(h \rightarrow e_{a} e_{b}\right) \equiv \Gamma\left(h \rightarrow e_{a}^{-} e_{b}^{+}\right)+\Gamma\left(h \rightarrow e_{a}^{+} e_{b}^{-}\right)=\frac{m_{h}}{8 \pi}\left(\left|\Delta_{(a b) L}\right|^{2}+\left|\Delta_{(a b) R}\right|^{2}\right) .
$$


Table 1. Couplings relating with decays $h \rightarrow e_{a} e_{b}$ in the E331X $X_{R}$ model. All momenta in the Feynman rules corresponding to these vertices are incoming.

\begin{tabular}{|c|c|}
\hline Vertex & Factor \\
\hline$h \overline{e_{a}} e_{a}$ & $-\frac{i g m_{a}}{2 m_{W}} c_{\xi}$ \\
\hline$h \overline{n_{i}} n_{j}$ & $-\frac{i g c_{\xi}}{2 m_{W}}\left(\lambda_{i j}^{0} P_{L}+\lambda_{i j}^{0 *} P_{R}\right)$ \\
\hline$H_{2}^{-} \overline{e_{a}} n_{i}, H_{2}^{+} \overline{n_{i}} e_{b}$ & $-\frac{i g c_{\alpha}}{\sqrt{2} m_{W}}\left(\lambda_{a i}^{L *} P_{R}+\lambda_{a i}^{R *} P_{L}\right),-\frac{i g c_{\alpha}}{\sqrt{2} m_{W}}\left(\lambda_{b i}^{L} P_{L}+\lambda_{b i}^{R} P_{R}\right)$ \\
\hline$W_{\mu}^{+} e_{b} \overline{n_{i}}, W_{\mu}^{-} \overline{e_{a}} n_{i}$ & $\frac{i g}{\sqrt{2}} U_{b i}^{v} \gamma^{\mu} P_{L}, \frac{i g}{\sqrt{2}} U_{a i}^{\nu *} \gamma^{\mu} P_{L}$ \\
\hline$V_{\mu}^{+} \overline{n_{i}} e_{b}, V_{\mu}^{-} n_{i} \overline{e_{a}}$ & $\frac{i g}{\sqrt{2}} U_{(b+3) i}^{v} \gamma^{\mu} P_{L}, \frac{i g}{\sqrt{2}} U_{(a+3) i}^{v *} \gamma^{\mu} P_{L}$ \\
\hline$h W^{+\mu} W^{-v}$ & $i g c_{\xi} m_{W} g_{\mu \nu}$ \\
\hline$h V^{+\mu} V^{-v}$ & $i g m_{V} g_{\mu v} \sin (\alpha-\xi)$ \\
\hline$H_{2}^{+} h V_{\mu}^{-}, V_{\mu}^{+} H_{2}^{-} h$ & $\frac{i g}{2}\left(P_{H_{2}^{+}}-P_{h}\right)_{\mu} \cos (\alpha-\xi), \frac{i g}{2}\left(P_{h}-P_{H_{2}^{-}}\right)_{\mu} \cos (\alpha-\xi)$ \\
\hline$h H_{2}^{+} H_{2}^{-}$ & $i \lambda_{H_{2}}^{0 \pm}=i c_{\alpha}^{2} c_{\xi}\left[2 \lambda_{2} v-\left(\lambda_{3}+\lambda_{4}\right) t_{\xi} w\right]$ \\
\hline
\end{tabular}

The corresponding branching ratio is $\operatorname{Br}\left(h \rightarrow e_{a} e_{b}\right)=\Gamma\left(h \rightarrow e_{a} e_{b}\right) / \Gamma_{h}^{\text {total }}$, where $\Gamma_{h}^{\text {total }} \simeq$ $4.1 \times 10^{-3} \mathrm{GeV}$ is the total decay width $[8,10]$. The $\Delta_{L, R}$ are scalar factors written as

$$
\Delta_{L, R}=\sum_{i=1,5,7,8} \Delta_{L, R}^{(i) W}+\sum_{i=1}^{10} \Delta_{L, R}^{(i) Y}
$$

All private factors $\Delta_{L, R}^{(i) W}$ and $\Delta_{L, R}^{(i) Y}$ denote one-loop contributions from Feynman diagram in Fig. 1. Analytic expressions are computed using techniques presented in [11-13]. The results are listed in the appendix.

At one-loop level, the Feynman diagrams contributing to the decay $\mu \rightarrow e \gamma$ are shown in Fig. 2.

The formula of $\operatorname{Br}(\mu \rightarrow e \gamma)$ is [14]

$$
\operatorname{Br}(\mu \rightarrow e \gamma)=\frac{12 \pi^{2}}{G_{F}^{2}}\left|D_{R}\right|^{2},
$$

where $G_{F}=g^{2} /\left(4 \sqrt{2} m_{W}^{2}\right)$ and $D_{R}=D_{R}^{W}+D_{R}^{V}+D_{R}^{H_{2}^{ \pm}}$,

$$
\begin{aligned}
D_{R}^{W} & =-\frac{e g^{2}}{32 \pi^{2} m_{W}^{2}} \sum_{j=1}^{9} U_{1 j}^{\nu *} U_{2 j}^{v} F\left(t_{j W}\right), \quad D_{R}^{V}=-\frac{e g^{2}}{32 \pi^{2} m_{V}^{2}} \sum_{j=1}^{9} U_{4 j}^{v *} U_{5 j}^{v} F\left(t_{j V}\right), \\
D_{R}^{H_{2}^{ \pm}} & =-\frac{e g^{2} c_{\theta}^{2}}{16 \pi^{2} m_{W}^{2}} \sum_{j=1}^{9}\left[\frac{\lambda_{1 j}^{L *} \lambda_{2 j}^{L}}{m_{H_{2}^{ \pm}}^{2}} \times \frac{1-6 t_{j H}+3 t_{j H 2}^{2}+2 t_{j H}^{3}-6 t_{j H}^{2} \ln \left(t_{j H}\right)}{12\left(t_{j H}-1\right)^{4}}\right. \\
& \left.+\frac{m_{n_{j}} \lambda_{1 j}^{L *} \lambda_{2 j}^{\prime R}}{m_{H_{2}^{ \pm}}^{2}} \times \frac{-1+t_{j H}^{2}-2 t_{j H} \ln \left(t_{j H}\right)}{2\left(t_{j H}-1\right)^{3}}\right]
\end{aligned}
$$




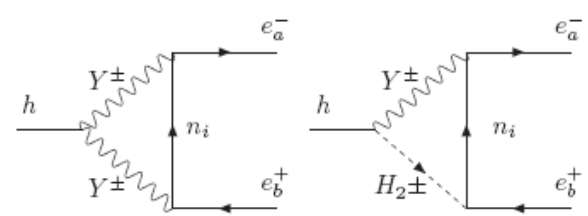

(1)

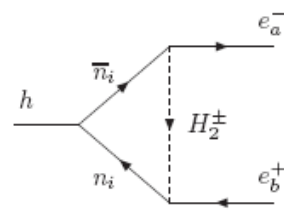

(6)
(2)

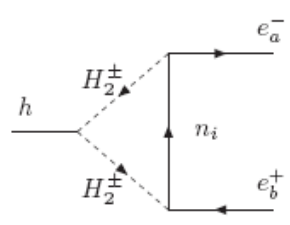

(7)

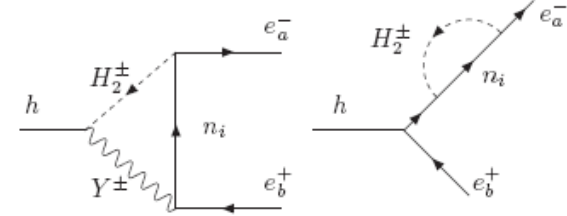

(3)

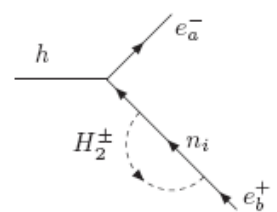

(5)

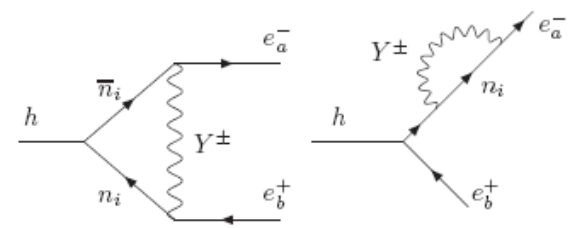

(8)

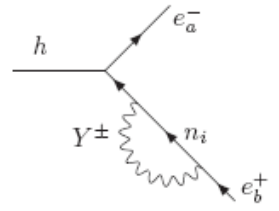

(10)

Fig. 1. One-loop Feynman diagrams contributing to the decay $h \rightarrow e_{a} e_{b}$ in the unitary gauge. Here $Y^{ \pm}=\left(W^{ \pm}, V^{ \pm}\right)$.

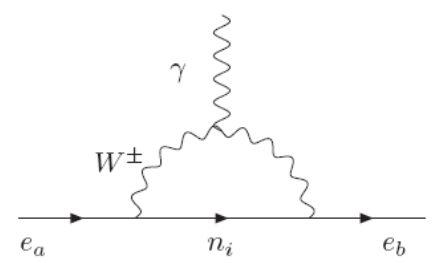

(1)

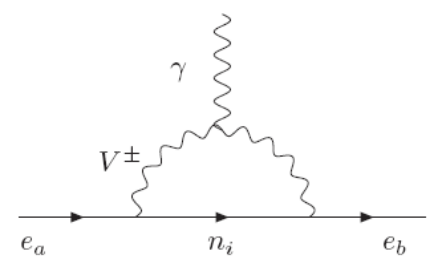

(2)

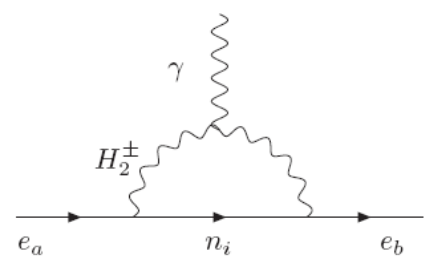

(3)

Fig. 2. Feynman diagrams for $\mu \rightarrow e \gamma$ at one-loop level in the unitary gauge.

where $t_{j W} \equiv \frac{m_{n_{j}}^{2}}{m_{W}^{2}}, t_{j V} \equiv \frac{m_{n_{j}}^{2}}{m_{V}^{2}}, t_{j H} \equiv \frac{m_{n_{j}}^{2}}{m_{H_{2}^{ \pm}}^{2}}$, and

$$
F(x) \equiv-\frac{10-43 x+78 x^{2}-49 x^{3}+4 x^{4}+18 x^{3} \ln (x)}{12(x-1)^{4}} .
$$

In the next section, we will focus on the allowed regions satisfying the upper bound of $\operatorname{Br}(\mu \rightarrow e \gamma)$ and giving large $\operatorname{Br}(h \rightarrow \mu \tau)$.

\section{NUMERICAL RESULT}

For numerical investigation, we choose a set of independent parameters as follows. The $S U(3)_{L}$ scale $w$ is equivalent to the heavy charged boson mass $m_{V}=g \sqrt{v^{2}+w^{2}} / 2$; the singly charged Higgs mass $m_{H_{2}^{ \pm}}$; the Higgs self-coupling factor $\lambda_{1}$; the mixing parameter $\xi$ between 
neutral Higgs bosons; heavy neutrino masses $m_{n_{4,5,6}}^{2}$; the parameters $z$ of $m_{D}$ matrix, and the matrix $M_{R}$.

Some parameters are the well-known experimental values [8]: the mass of $W$ boson $m_{W}=$ $80.385 \mathrm{GeV}$; charged lepton masses: $m_{e}=5.10^{-4} \mathrm{GeV}, m_{\mu}=0.105 \mathrm{GeV}, m_{\tau}=1.776 \mathrm{GeV} ; m_{h}=$ $125.1 \mathrm{GeV}$; the $S U(2)_{L}$ gauge coupling $g=0.651$, and $v \simeq 246 \mathrm{GeV}$.

Other parameters are functions of the independent ones. Specifically, the mixing angle $\alpha$ and Higgs self couplings $\lambda_{4}$ are fixed by the relations (10) and (3), respectively. The Higgs couplings $\lambda_{2,3}$ are written as functions of $\xi$ and $m_{h}$. The parameters $x_{1,2}$ and active neutrino masses in $m_{D}$ are exactly the same as those mentioned in Ref. [7].

For simplicity and without losing generality, we choose $M_{R}=M_{R} I_{3}$ with $M_{R}=k z$, where $k$ will be used. In numerical calculation, default values of free parameters are chosen as $\lambda_{1}=1$, $m_{Y}=1.5 \mathrm{TeV}, c_{\xi}=0.99, \mu_{X}=10^{-7} \mathrm{GeV}$. We note that the recent experimental data $h \rightarrow \gamma \gamma$ gives $\left|c_{\xi}\right| \geq 0.99$. The perturbative limit of Yukawa coupling of lepton gives $z \leq 600 \mathrm{GeV}$. According to Ref. [7], in order to produce active neutrino masses and mixing which can be fitted to recent experimental data, we need a requirement that $k \geq 5.5$.

We checked numerically in the regions with large $k \geq 500$ and $z=1,5,10,500 \mathrm{GeV}$, the results completely agree with [6,7]. Furthermore, all allowed regions satisfying $\operatorname{Br}(\mu \rightarrow e \gamma) \leq$ $4.2 \times 10^{-13}$ also give a small $B r(h \rightarrow \mu \tau) \leq 10^{-9}$. These regions are not interesting, we will not pay attention to them any more.

For illustration with small $k$, we will survey with two cases of $k=6$ and $k=9$ and different allowed values of $z=(50,100,300,500,600) \mathrm{GeV}$. As shown in figure 3 , the results are very positive, where there exist narrow regions satisfying $\operatorname{Br}(\mu \rightarrow e \gamma) \leq 4.2 \times 10^{-13}$. It can be explained
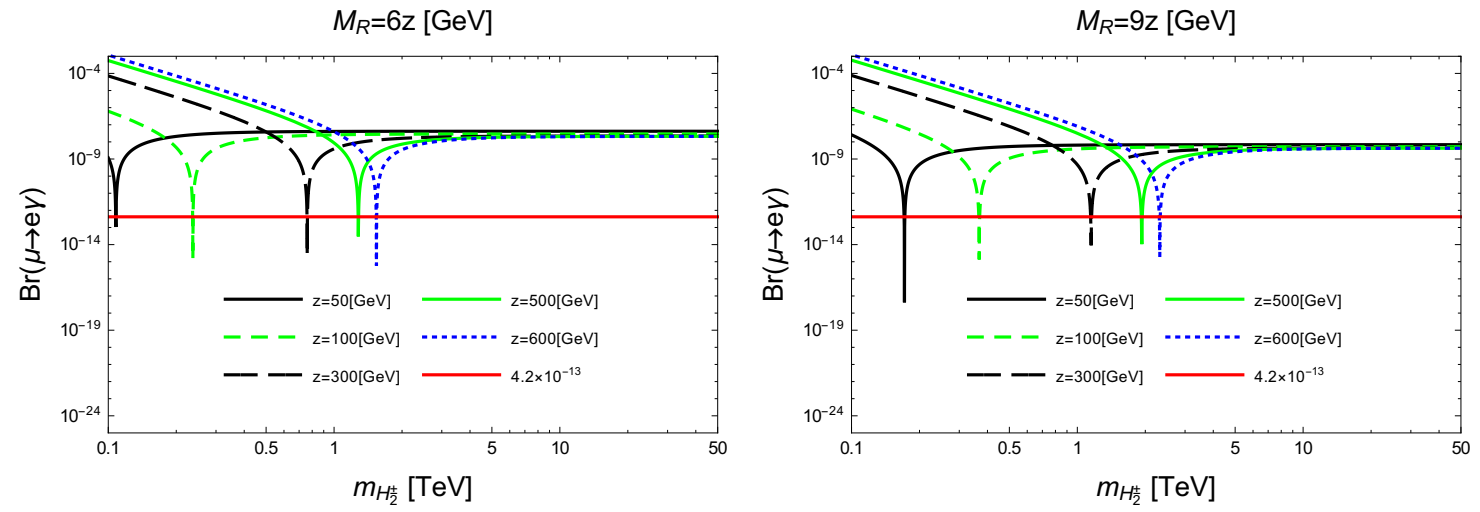

Fig. 3. $\operatorname{Br}(\mu \rightarrow e \gamma)$ as function of $m_{H_{2}}^{ \pm}$.

by the destructive correlations of the gauge and Higgs contributions. These properties does not appear in the ISS version of the SM, where charged Higgs bosons do not appear. The effects of $k$ on the allowed regions can be seen in Fig. 4, where larger $k$ will result in wider allowed regions.

To continue, we will investigate the $\operatorname{Br}(h \rightarrow \mu \tau)$ in regions with small $k$ and different values of $z$ similarly to the case of $\operatorname{Br}(\mu \rightarrow e \gamma)$. The results are shown in Fig. 5. $\operatorname{The} \operatorname{Br}(h \rightarrow \mu \tau)$ is enhanced strongly with increasing $z$, and changes slowly with $m_{H_{2}^{ \pm}} \operatorname{Br}(h \rightarrow \mu \tau)$ can reach values 

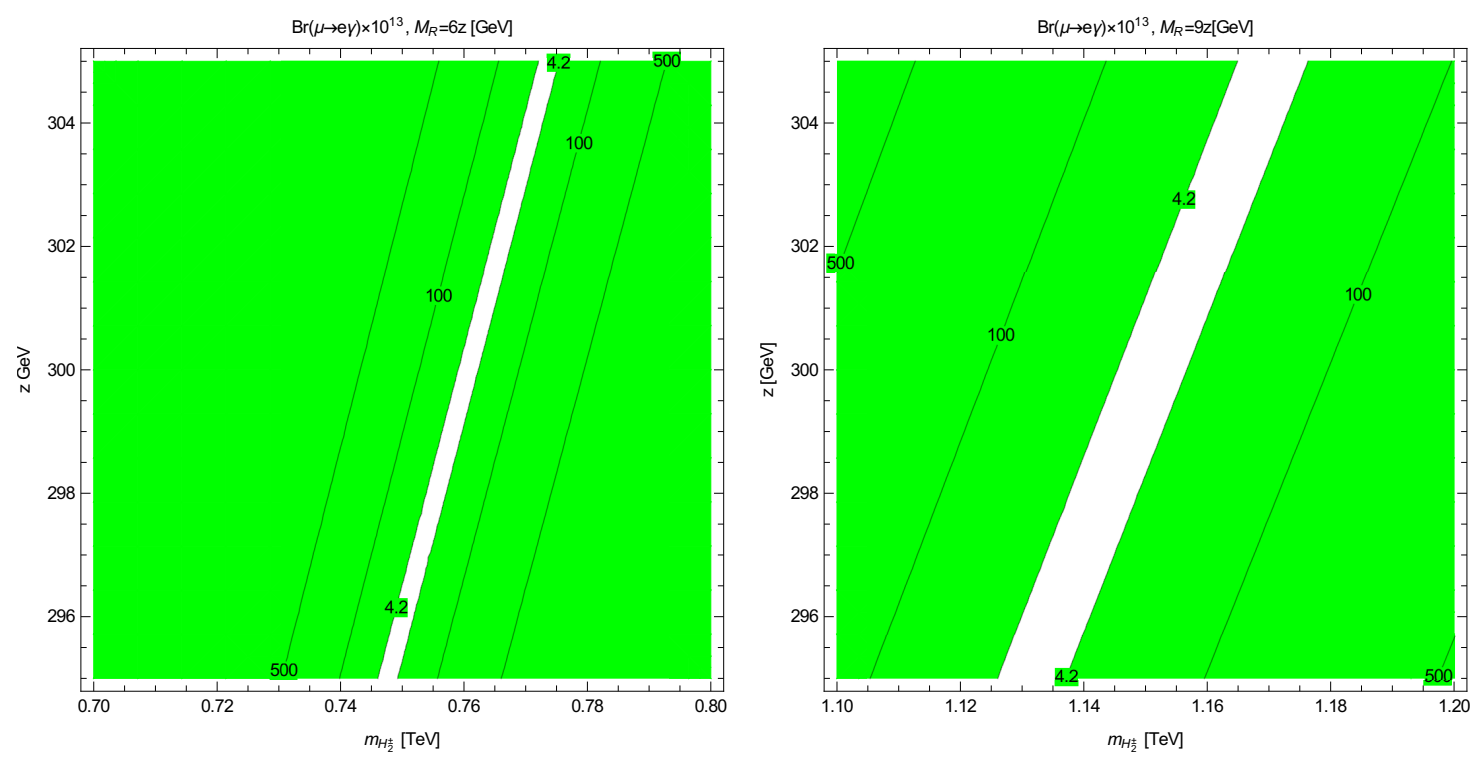

Fig. 4. Contours plots of $\operatorname{Br}(\mu \rightarrow e \gamma)$ as functions of $z$ and $m_{H_{2}^{ \pm}}^{2}$. The green regions are excluded by the recent experimental data $\operatorname{Br}(\mu \rightarrow e \gamma) \leq 4.2 \times 10^{-13}$.
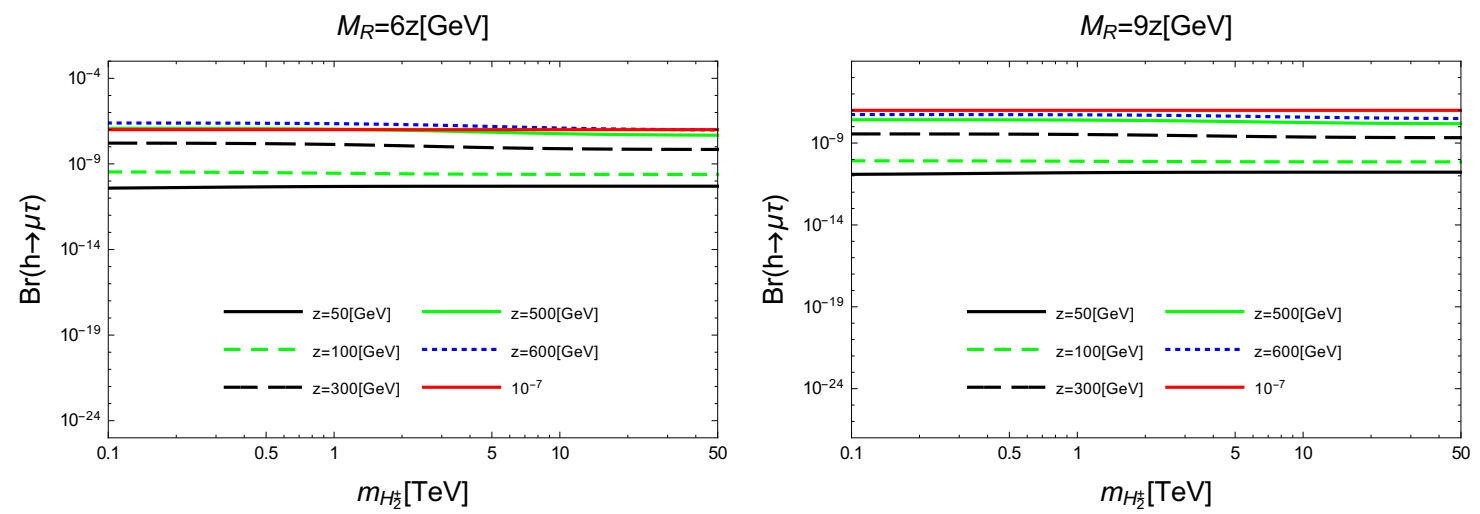

Fig. 5. Branching ratio of $h \rightarrow \mu \tau$ as function of $m_{H_{2}^{ \pm}}$with two case $k=6$ (left panel) and $k=9$ (right panel).

of $\mathscr{O}\left(10^{-7}\right)$, in case $k=6$ and $m_{H_{2}^{ \pm}}$is between $0.1-5 \mathrm{TeV}$. With all values of $z$ in the perturbative limit $z \leq 600 \mathrm{GeV}$, there always exist allowed regions predicting small ranges of $m_{H_{2}^{ \pm}}$.

The results of two specific cases $k=6, k=9$ and $z=300 ; 500 ; 600 \mathrm{GeV}$ are given in Table 2 . This is a very interesting prediction of the $\mathrm{E} 331 X_{R}$, where both of the $\operatorname{Br}(h \rightarrow \mu \tau)$ and $(\mu \rightarrow e \gamma)$ can reach the detection of the future experiments. These results are also consistent with previous publications such as $[7,14]$. They are more interesting than those mentioned in [6], where only large $\operatorname{Br}(\mu \rightarrow e \gamma)$ was shown in the case of large $k$. 
Table 2. Values of $\operatorname{Br}(h \rightarrow \mu \tau)$ with some fixed $k$ and $z$.

\begin{tabular}{|c|c|c|c|}
\hline$k$ & $z[\mathrm{GeV}]$ & $\begin{array}{c}\text { Value of } m_{H_{2}^{ \pm}}[\mathrm{TeV}] \\
\text { satisfying } \operatorname{Br}(\mu \rightarrow e \gamma) \leq 4.2 \times 10^{-13}\end{array}$ & $\operatorname{Br}(h \rightarrow \mu \tau)$ \\
\hline 6 & 300 & {$[0.759 ; 0.762]$} & {$[1.41 ; 1.44] \times 10^{-8}$} \\
\hline 9 & 300 & {$[1.146 ; 1.156]$} & {$[3.01 ; 3.30] \times 10^{-9}$} \\
\hline 6 & 500 & {$[1.280 ; 1.282]$} & {$[1.024 ; 1.025] \times 10^{-7}$} \\
\hline 9 & 500 & {$[1.926 ; 1.933]$} & {$[2.398 ; 2.400] \times 10^{-8}$} \\
\hline 6 & 600 & {$[1.540 ; 1.542]$} & {$[2.1002 ; 2.1007] \times 10^{-7}$} \\
\hline 9 & 600 & {$[2.314 ; 2.323]$} & {$[4.960 ; 4.963] \times 10^{-8}$} \\
\hline
\end{tabular}

\section{CONCLUSIONS}

In this paper we have shown that the $\mathrm{E} 331 X_{R}$ predicts large $\mathrm{Br}$ of lepton flavor violation, especially in the regions having large $m_{D}$ but light exotic neutrinos. Similarly to previous investigation of other 3-3-1 models with inverse seesaw neutrino, the region with very heavy exotic neutrino $(k \gg 1)$, the model predicts that $\operatorname{Br}(\mu \rightarrow e \gamma)$ can reach recent upper bound of experiment. In contrast, the $\operatorname{Br}(h \rightarrow \mu \tau)<\mathscr{O}\left(10^{-9}\right)$, which is still too small for searching this signal. For small $k \geq 6$, there exist very narrow regions satisfying experimental bound of $\operatorname{Br}(\mu \rightarrow e \gamma)$ and predict large $\operatorname{Br}(h \rightarrow \mu \tau)$, which can be larger than $\mathscr{O}\left(10^{-7}\right)$. Furthermore, $m_{H_{2}^{ \pm}}$is in order of $\mathscr{O}(1) \mathrm{TeV}$. In the region with small $k$, the appearance of $H_{2}^{ \pm}$is very important. Because the allowed regions, which have very small $\operatorname{Br}(\mu \rightarrow e \gamma)$, arise from the destructive correlations of charged Higgs and gauge boson contributions to the $\operatorname{Br}(\mu \rightarrow e \gamma)$.

The LFV decay rates of the two processes $\mu \rightarrow e \gamma$ and $h \rightarrow \mu \tau$ are suppressed in the E331 models. Hence, these two channel decays will give important information to distinguish the E331 models and the $\mathrm{E} 331 X_{R}$ if any LFV signals are detected in the future.

\section{ACKNOWLEDGMENT}

This research is funded by the Vietnam National Foundation for Science and Technology Development (NAFOSTED) under the grant number 103.01-2017.29.

\section{REFERENCES}

[1] W. A. Ponce, Y. Giraldo and L. A. Sanchez, Phys. Rev. D67 (2003) 075001.

[2] P. V. Dong, H. N. Long, D. T. Nhung and D. V. Soa, Phys. Rev. D73 (2006) 035004.

[3] R. Foot, H. N. Long and T. A. Tran, Phys. Rev. D50 (1994) R34.

[4] A. M. Baldini et al., Eur. Phys. J. C76 (2016) 434.

[5] A. M. Sirunyan et al., JHEP 06 (2018) 001.

[6] S. M. Boucenna, J. W. F. Valle and A. Vicente, Phys. Rev. D92 (2015) 053001.

[7] T. P. Nguyen, T. T. Le, T. T. Hong and L. T. Hue, Phys. Rev. D97 (2018) 073003.

[8] C. Patrignani et al., Chin. Phys. C40 (2016) 100001.

[9] L. T. Hue, H. N. Long, T. T. Thuc and T. Phong Nguyen, Nucl. Phys. B907 (2016) 37.

[10] A. Denner, S. Heinemeyer, I. Puljak, D. Rebuzzi and M. Spira, Eur. Phys. J. C71 (2011) 1753.

[11] T. T. Thuc, L. T. Hue, H. N. Long and T. P. Nguyen, Phys. Rev. D93 (2016) 115026. 
[12] N. H. Thao, L. T. Hue, H. T. Hung and N. T. Xuan, Nucl. Phys. B921 (2017) 159.

[13] K. H. Phan, H. T. Hung and L. T. Hue, PTEP 2016 (2016) 113 B03.

[14] L. T. Hue, L. D. Ninh, T. T. Thuc and N. T. T. Dat, Eur. Phys. J. C78 (2018) 128.

\section{APPENDIX}

Here we only give analytic expressions for contribution from Feynman diagrams $(4),(5),(6),(7)$ in Fig. 1 with charged Higgs bosons $\left(H_{2}^{ \pm}\right)$. The remaining diagrams are calculated in the same way.

$$
\begin{aligned}
& \Delta_{L}^{(4+5) V H_{2}^{ \pm}}=-\frac{g^{3} c_{\alpha} c_{\zeta}^{2}}{32 \pi^{2} m_{W}^{3}\left(m_{a}^{2}-m_{b}^{2}\right)} \\
& \times \sum_{i=1}^{9}\left[m_{a} m_{b} m_{n_{i}} \lambda_{a i}^{L *} \lambda_{b i}^{R}\left(B_{0}^{(1)}-B_{0}^{(2)}\right)+m_{n_{i}} \lambda_{a i}^{R *} \lambda_{b i}^{L}\left(m_{b}^{2} B_{0}^{(1)}-m_{a}^{2} B_{0}^{(2)}\right)\right. \\
& \left.+m_{a} m_{b}\left(\lambda_{a i}^{L *} \lambda_{b i}^{L} m_{b}+\lambda_{a i}^{R *} \lambda_{b i}^{R} m_{a}\right)\left(B_{1}^{(1)}+B_{1}^{(2)}\right)\right], \\
& \Delta_{R}^{(4+5) V H_{2}^{ \pm}}=-\frac{g^{3} c_{\alpha} c_{\zeta}^{2}}{32 \pi^{2} m_{W}^{3}\left(m_{a}^{2}-m_{b}^{2}\right)} \\
& \times \sum_{i=1}^{9}\left[m_{a} m_{b} m_{n_{i}} \lambda_{a i}^{R *} \lambda_{b i}^{L}\left(B_{0}^{(1)}-B_{0}^{(2)}\right)+m_{n_{i}} \lambda_{a i}^{L *} \lambda_{b i}^{R}\left(m_{b}^{2} B_{0}^{(1)}-m_{a}^{2} B_{0}^{(2)}\right)\right. \\
& \left.+m_{a} m_{b}\left(\lambda_{a i}^{R *} \lambda_{b i}^{R} m_{b}+\lambda_{a i}^{L *} \lambda_{b i}^{L} m_{a}\right)\left(B_{1}^{(1)}+B_{1}^{(2)}\right)\right], \\
& \Delta_{L}^{(6) V H_{2}^{ \pm}}=-\frac{g^{3} c_{\alpha} c_{\zeta}^{2}}{32 \pi^{2} m_{W}^{3}} \sum_{i, j=1}^{9}\left\{\lambda _ { i j } ^ { 0 * } \left[\lambda_{a i}^{R *} \lambda_{b j}^{L}\left(B_{0}^{(12)}+m_{H_{k}^{ \pm}}^{2} C_{0}-m_{a}^{2} C_{1}+m_{b}^{2} C_{2}\right)\right.\right. \\
& \left.+\lambda_{a i}^{R *} \lambda_{b j}^{R} m_{b} m_{n_{j}} C_{2}-\lambda_{a i}^{L *} \lambda_{b j}^{L} m_{a} m_{n_{i}} C_{1}\right] \\
& +\lambda_{i j}^{0}\left[\lambda_{a i}^{R *} \lambda_{b j}^{L} m_{n_{i}} m_{n_{j}} C_{0}+\lambda_{a i}^{R *} \lambda_{b j}^{R} m_{n_{i}} m_{b}\left(C_{0}+C_{2}\right)\right. \\
& \left.\left.+\lambda_{a i}^{L *} \lambda_{b j}^{L} m_{a} m_{n_{j}}\left(C_{0}-C_{1}\right)+\lambda_{a i}^{L *} \lambda_{b j}^{R} m_{a} m_{b}\left(C_{0}-C_{1}+C_{2}\right)\right]\right\}, \\
& \Delta_{R}^{(6) V H_{2}^{ \pm}}=-\frac{g^{3} c_{\alpha} c_{\zeta}^{2}}{32 \pi^{2} m_{W}^{3}} \sum_{i, j=1}^{9}\left\{\lambda _ { i j } ^ { 0 } \left[\lambda_{a i}^{L *} \lambda_{b j}^{R}\left(B_{0}^{(12)}+m_{H_{2}^{ \pm}}^{2} C_{0}-m_{a}^{2} C_{1}+m_{b}^{2} C_{2}\right)\right.\right. \\
& \left.+\lambda_{a i}^{L *} \lambda_{b j}^{L} m_{b} m_{n_{j}} C_{2}-\lambda_{a i}^{R *} \lambda_{b j}^{R} m_{a} m_{n_{i}} C_{1}\right] \\
& +\lambda_{i j}^{0 *}\left[\lambda_{a i}^{L *} \lambda_{b j}^{R} m_{n_{i}} m_{n_{j}} C_{0}+\lambda_{a i}^{L *} \lambda_{b j}^{L} m_{n_{i}} m_{b}\left(C_{0}+C_{2}\right)\right. \\
& \left.\left.+\lambda_{a i}^{R *} \lambda_{b j}^{R} m_{a} m_{n_{j}}\left(C_{0}-C_{1}\right)+\lambda_{a i}^{R *} \lambda_{b j}^{L} m_{a} m_{b}\left(C_{0}-C_{1}+C_{2}\right)\right]\right\}, \\
& \Delta_{L}^{(7) V H_{2}^{ \pm}}=\frac{g^{2} \lambda_{H_{k}}^{ \pm} c_{\zeta}^{2}}{16 \pi^{2} m_{W}^{2}} \sum_{i=1}^{9}\left[-\lambda_{a i}^{R *} \lambda_{b i}^{L} m_{n_{i}} C_{0}-\lambda_{a i}^{L *} \lambda_{b i}^{L} m_{a} C_{1}+\lambda_{a i}^{R *} \lambda_{b i}^{R} m_{b} C_{2}\right], \\
& \Delta_{R}^{(7) V H_{2}^{ \pm}}=\frac{g^{2} \lambda_{H_{2}}^{ \pm} c_{\zeta}^{2}}{16 \pi^{2} m_{W}^{2}} \sum_{i=1}^{9}\left[-\lambda_{a i}^{L *} \lambda_{b i}^{R} m_{n_{i}} C_{0}-\lambda_{a i}^{R *} \lambda_{b i}^{R} m_{a} C_{1}+\lambda_{a i}^{L *} \lambda_{b i}^{L} m_{b} C_{2}\right],
\end{aligned}
$$

